\title{
Plasmonic Band-Pass Microfilters for LWIR Absorption Spectroscopy
}

\author{
J. M. Banks, ${ }^{1}$ P. D. Flammer, ${ }^{1}$ T. E. Furtak, ${ }^{1}$ R. E. Hollingsworth, ${ }^{2}$ and R. T. Collins ${ }^{1}$ \\ ${ }^{1}$ Department of Physics, Colorado School of Mines, 1500 Illinois Street, Golden, CO 80401, USA \\ ${ }^{2}$ Thin Film Technologies Division, ITN Energy Systems, Inc., 8130 Shaffer Parkway, Littleton, CO 80127, USA \\ Correspondence should be addressed to P. D. Flammer, pflammer@mines.edu
}

Received 31 October 2011; Revised 13 January 2012; Accepted 13 January 2012

Academic Editor: K. S. Chiang

Copyright (C) 2012 J. M. Banks et al. This is an open access article distributed under the Creative Commons Attribution License, which permits unrestricted use, distribution, and reproduction in any medium, provided the original work is properly cited.

\begin{abstract}
Absorption spectroscopy in the long wave infrared provides an effective method for identification of various hazardous chemicals. We present a theoretical design for plasmonic band-pass filters that can be used to provide wavelength selectivity for uncooled microbolometer sensors. The microfilters consist of a pair of input reflection gratings that couple light into a plasmonic waveguide with a central resonant waveguide cavity. An output transmission grating on the other side of the structure pulls light out of the waveguide where it is detected by a closely spaced sensor. Fabrication of the filters can be performed using standard photolithography procedures. A spectral bandpass with a full-width at half-maximum (FWHM) of $100 \mathrm{~nm}$ can be obtained with a center wavelength spanning the entire $8-12 \mu \mathrm{m}$ atmospheric transmission window by simple geometric scaling of only the lateral dimensions. This allows the simultaneous fabrication of all the wavelength filters needed for a full spectrometer on a chip.
\end{abstract}

\section{Introduction}

Detection of long-wave infrared (LWIR) light in the $8-12 \mu \mathrm{m}$ atmospheric transmission band is an important area of research with a high demand for good wavelength resolution, high sensitivity, portability, and affordability. An important application for LWIR detection is absorption spectroscopy for remote sensing of chemical vapors [1]. There have been several approaches for spectral imaging of LWIR light. One approach is to rapidly tune a liquid crystal-filled Fabry-Perot etalon coupled with a cooled LWIR camera to detect light over a wide wavelength range with good spectral resolution [2]. Cryogenic cooling, however, greatly increases the cost of operation and portability of the detector. Uncooled microbolometer sensors are commonly used as an alternative to the cooled detectors for thermal imaging, but they show low sensitivity [3]. It has been shown in recent work [4] that uncooled microbolometers, absorbing only a narrow band of radiation, can have sensitivities approaching that of cooled detectors. There are several ways to narrow the band of absorbed light in a microbolometer detector, including antennas [5-7], metamaterials [8-11], and vertical optical cavities
[4, 12-17]. Vertical Fabry-Perot cavities are especially convenient to use since they can be placed above conventional microbolometer arrays. However, this requires the vertical dimensions to be changed for the detection of different wavelengths. The most common way this is achieved is by tuning the resonant wavelength by moving one of the mirrors via an included microelectromechanical system (MEMS) element. However, this scheme tends to be vibrationally sensitive and requires a high bias voltage. Surface plasmon supporting structures, involving metal gratings, have also been exploited to enhance the signal-to-noise ratio of an integrated detector [18]. In that design $\mathrm{Yu}$ et al. demonstrated a bandpass with FWHM of less than $200 \mathrm{~nm}$ in a structure that could be optimized for wavelengths between 7 and $11 \mu \mathrm{m}$. However, the integrated nature of their device would require fabrication of the detector as part of the structure. Also, for spectroscopic applications, high spectral resolution is necessary. The vertical Fabry-Perot resonators discussed above have bandwidths of $150 \mathrm{~nm}$ or larger $[2,8,12]$.

Here, we present finite-element simulations of plasmonic bandpass microfilters with a target bandwidth of 50-100 nm that can be combined with a conventional microbolometer 


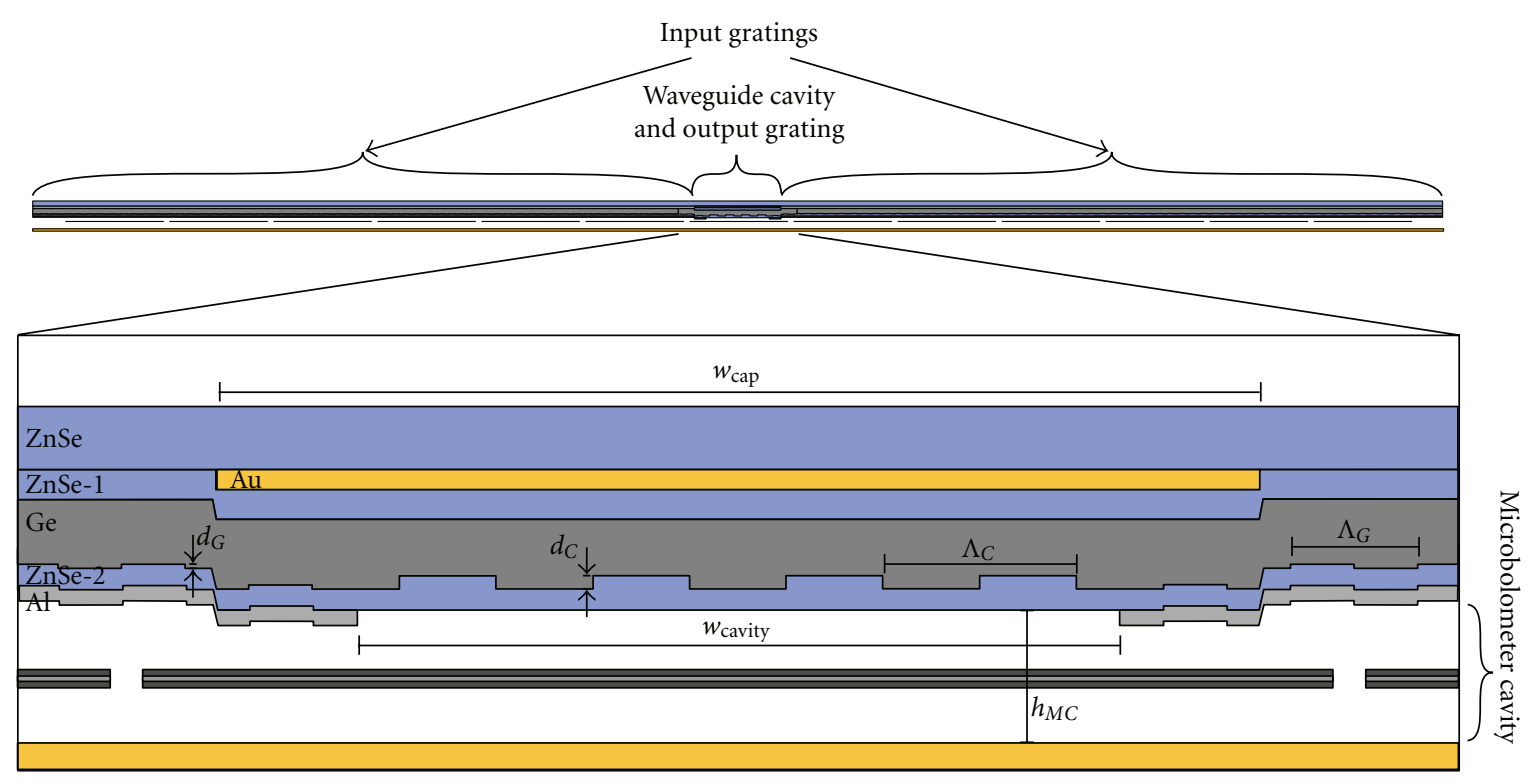

FIGURE 1: Schematic of wavelength filter and microbolometer array. The upper view shows the entire structure to the extent of the input gratings. The inset, shows the waveguide cavity, microbolometer cavity, and the first few periods of the input grating. The first two periods of the input gratings can be seen on the left and right edges of the inset and the four periods of the output grating can be seen in the center of the inset.

array. A set of adjacent microfilters with different center wavelengths would convert the microbolometer array into a spectrometer. The center wavelength in our design can be adjusted over the full LWIR band by changing only horizontal dimensions, allowing a full spectrometer on a chip to be constructed in a single set of 4 lithography steps. Since there are no moving parts, this is a design that is vibrationally insensitive and simple to manufacture while maintaining the high sensitivity and low noise of a Fabry-Perot cavity.

\section{Description of the Structure}

The microfilter structure consists of two input gratings on either side of a central waveguide cavity with an output grating on the opposite side that has a cavity to direct optical energy into the microbolometer detector. A schematic of the microfilter is shown in Figure 1.

Light is incident from the top, through a zinc selenide substrate and is grating coupled into a plasmonic waveguide consisting of a deposited $\mathrm{ZnSe}$ layer (ZnSe-1 in the figure) of thickness $t_{\mathrm{ZnSe}, 1}$, a germanium layer of thickness $t_{\mathrm{Ge}}$, a $\mathrm{ZnSe}$ layer ( $\mathrm{ZnSe}-2$ in the figure) of thickness $t_{\mathrm{ZnSe}, 2}$, and an aluminum layer of thickness $t_{\mathrm{Al}}$. ZnSe was used as a substrate to index match the ZnSe-1 layer, but the design could easily be adapted to support lower-cost silicon wafer substrates. This can be achieved by depositing a thick enough $\mathrm{ZnSe-1}$ layer on a silicon substrate that only a small amount of field penetrates the substrate. ZnSe-1 thicknesses as low as $2.5 \mu \mathrm{m}$ shows field penetration of only $1 \%$. The lower surface of the germanium has an input grating with $N_{G}$ grooves of depth $d_{G}$. These four layers form a hybrid plasmon/dielectric slab waveguide, where the grating couples light at certain resonant wavelengths into the waveguide and reflects offresonance wavelengths. Because of the plasmonic component, the input grating strongly selects TM polarization. The grating depth and number of grooves control the bandwidth and the efficiency of coupling of light into the waveguide. The thicknesses of germanium and ZnSe-2 control the effective mode index of the waveguide. These parameters can be adjusted to affect the coupling efficiency and ohmic losses. In order to allow for easy and affordable fabrication of microfilter arrays, it is important that filters for different wavelengths can be manufactured with the same vertical dimensions, allowing all pixels to be fabricated using the same lithography steps. Therefore, in our simulations, vertical dimensions were initially optimized for operation near $10 \mu \mathrm{m}$ and then held constant for all the structures discussed in this paper. The input grating periodicity $\left(\Lambda_{G}\right)$ can be adjusted without extra lithography steps and was therefore used to select the resonant wavelength.

Once coupled into the waveguide via the input grating, light travels into a central waveguide cavity of width $w_{\text {cavity }}$. A lateral Fabry-Perot resonance is set up with the gratings acting as mirrors and the gap between the gratings as the cavity [19]. The cavity resonance increases the $Q$ (reduces FWHM) and also provides additional filtering of unfiltered light. A metal cap of thickness $t_{\text {cap }}$ located over the cavity area, of width $w_{\text {cap }}$, prevents direct transmission of undesired wavelengths. An opening in the aluminum layer under the cavity provides an exit path for light to reach the detector. An output coupling grating, with $N_{C}$ grooves of depth $d_{C}$, would be etched into the germanium layer of the cavity. The cavity groove depth not only adjusts the coupling efficiency for light coming out of the cavity, but also decreases the quality of the cavity resonance. The width of the cavity $\left(w_{C}\right)$ and 
the periodicity of the cavity grating $\left(\Lambda_{C}\right)$ can be adjusted to provide additional wavelength selectivity. This is possible since Fabry-Perot etalon theory states that the transmitted wavelength is dependent on the cavity length. Also, the resonant wavelength of the grating is dependent on the periodicity of the grating.

Once the light leaves the cavity, it enters the region containing the microbolometer detector array. The filters can be fabricated on the inside of the Si wafer lid used during wafer level vacuum packaging [13], providing a vibrationally insensitive, fixed spacing between the filter and microbolometer pixels. One filter structure covers about 11 microbolometers in the array, each microbolometer being $45 \mu \mathrm{m}$ wide. To provide the maximum flexibility in cavity size while illuminating a single pixel, an early-generation focal plane array geometry with $45 \mu \mathrm{m}$ pixels with $2 \mu \mathrm{m}$ gaps was used, but the results indicate that smaller pixel sizes as described in [20] or [21] could be used equally well. A continuous gold back reflector was incorporated into the model instead of the standard patterned aluminum reflector in order to minimize potential losses. Gold was expected to improve performance, but, since both aluminum and gold are very good metals in this wavelength range, the structure was insensitive to the type of metal in the reflector. The microbolometer pixels were placed $2 \mu \mathrm{m}$ above the back reflector in order to form a quarter wave resonant cavity for the LWIR band. The simulated microbolometer pixel included only SiN/a-SiGe/SiN layers without the broad band metal absorber typically included in imaging arrays. The metal absorber was found to be unnecessary in our design because the deposited SiN has an absorption band spanning the LWIR region of interest. The vertical position of the back reflector $\left(h_{M}\right)$ to the filter was adjusted to maximize coupling into the microbolometer. Note that our 2D simulation assumes a long dimension into the page of Figure 1. The structure will then lie on a line of microbolometers, where the response of the adjacent microbolometer pixels can be summed. The nearest neighbors in the line of microbolometers can be seen in Figure 1 as well.

\section{Results}

All 2D simulation was done using the commercial finite-element analysis package, COMSOL Multiphysics, which solves the frequency domain Helmholtz equation for the electric and magnetic fields. A mirror symmetry axis was used on the left boundary (center of structure) to reduce memory requirements, with all other boundaries truncated using perfectly matched layers [22]. Optical constants for ZnSe and metals were taken from the literature [23-25], while spectroscopic ellipsometry was used to measure optical constants of $\mathrm{Ge}$, SiGe, and SiN films grown using plasma-enhanced chemical vapor deposition in our labs. All measured optical constants are presented in the Appendix section. Three structures were simulated and optimized to detect light at wavelengths of $9,9.9$, and $11 \mu \mathrm{m}$, respectively. The steps in the optimization were as follows. First, the input grating periodicity was set to have a primary coupling resonance at the desired wavelength. Next, the cavity was optimized, and finally the vertical spacing between the filter and microbolometer array was optimized. Two figures of merit were calculated to describe the performance of the structures for the optimizations: transmitted power normalized to the incident power on the entire structure, and the spectral bandwidth as measured by the filter response Q (peak wavelength/ FWHM). There was often a tradeoff between these figures of merit, as discussed in Section 3.2.

3.1. Input Grating Optimization. Keeping the layer thicknesses fixed, the input grating periodicity was varied to study the effect on the peak wavelength. The layer thicknesses were fixed so that the structure can be fabricated with a single set of lithography steps. When the grating period is equal to integer values of the free-space wavelength divided by the effective index of the waveguide mode, the light is efficiently coupled into the waveguide. Therefore, if the layer thicknesses remain constant, the peak wavelength can be chosen by adjusting the grating periodicity. Figure 2 shows the coupling efficiency for incident light coming into the waveguide as a function of wavelength for five different grating periodicities. There is a linear relationship between grating periodicity and peak wavelength over the LWIR wavelength range $(8-12 \mu \mathrm{m})$. The relationship can be expressed as $\lambda_{0}=3.06 \Lambda_{C}+1.37$, where $\lambda_{0}$ (peak wavelength) and $\Lambda_{C}$ (grating periodicity) are both expressed in microns. This shows that the effective mode index is roughly constant over the LWIR band. Power is coupled equally in both directions because of the square grating profile, as can be seen in the inset of time average power flow.

This linear relationship will make designing different gratings for different wavelengths very simple. Because of higher-order couplings, gratings with larger peak wavelengths exhibit second-order peaks at smaller wavelengths. The second-order peaks for the gratings with periodicities of $3.14 \mu \mathrm{m}$ and $3.46 \mu \mathrm{m}$ can be seen at wavelengths of $8.2 \mu \mathrm{m}$ and $8.75 \mu \mathrm{m}$, respectively. It will be shown in Section 3.2 that these secondary peaks can be suppressed using a cavity and have little effect on the completed device.

The number of grooves was also optimized by varying the number of grooves while keeping the vertical dimensions the same. Figure 3 shows how the number of grooves affects coupling efficiency and quality factor. As the number of grooves increases, the quality factor continually increases, but the coupling efficiency reaches a maximum and eventually starts to decrease. As the number of grooves increases, the losses eventually overpower the benefit of a high number of grooves and causes the coupling efficiency to decrease. The two loss mechanisms that control this are ohmic losses in the metal and coupling of light back into free space. For the layer thicknesses used in this simulation, the number of grooves was chosen to be 80 for each grating. This produced a reasonable coupling efficiency of $6.8 \%$ and a high quality factor of 108 .

3.2. Waveguide Cavity Optimization. The optimal width of a smooth cavity would need to be an integral number of wavelengths. But with the grating as part of the waveguide core, the average mode index is perturbed. To find the best 


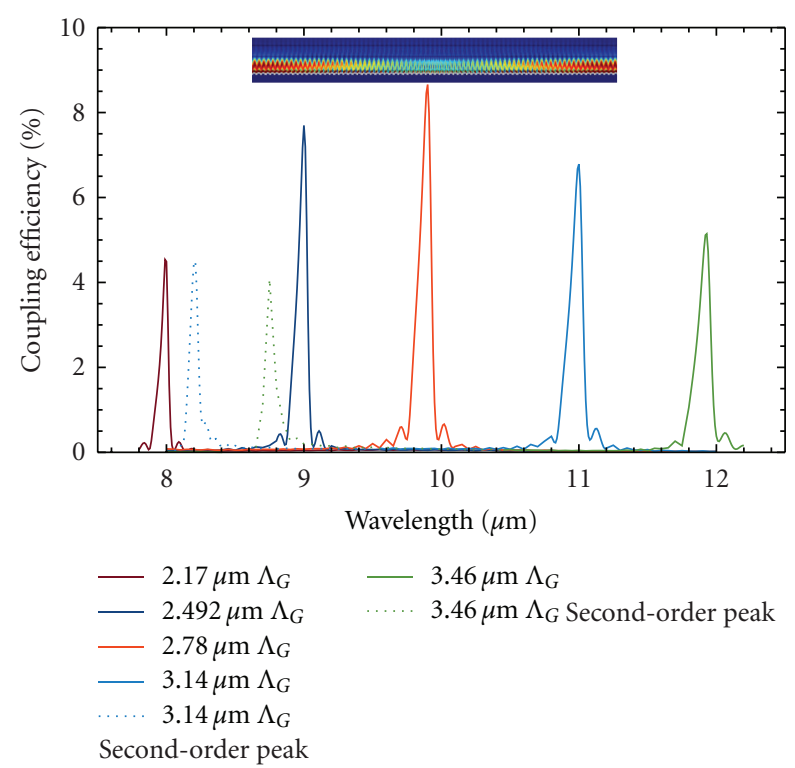

(a)

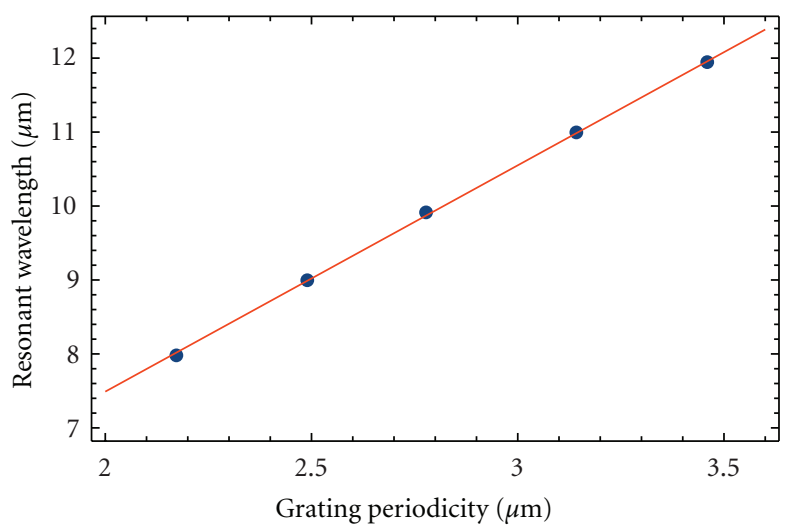

(b)

FIGURE 2: (a) Comparison of coupling efficiency spectra for several different grating periodicities. The coupling efficiency is defined as the fraction of the total incident power that leaves the grating in one direction. Dotted lines indicate second-order peaks. Inset is a plot of the time averaged power flow (magnitude of the Poynting vector) for a grating resonant at $11 \mu \mathrm{m}$. The aspect ratio is expanded vertically by $6 \mathrm{x}$ for better visibility. (b) Resonant wavelength for several different grating periodicities. Parameters used: $t_{\mathrm{Ge}}=1.65 \mu \mathrm{m}, t_{\mathrm{ZnSe}, 2}=200 \mathrm{~nm}$, $t_{\mathrm{Al}}=200 \mathrm{~nm}, d_{G}=100 \mathrm{~nm}, N_{G}=80$.

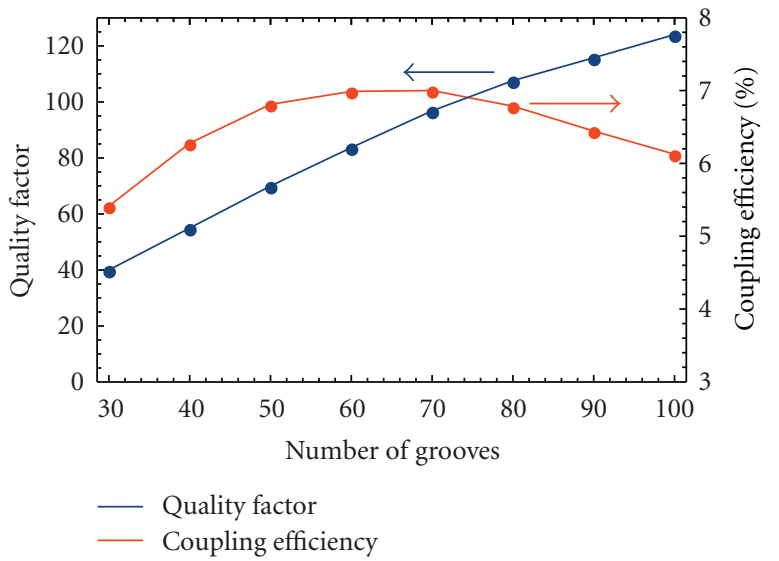

Figure 3: Comparison of coupling efficiency and quality factor as a function of number of grooves. Parameters used: $t_{\mathrm{Ge}}=1.65 \mu \mathrm{m}$, $t_{\mathrm{ZnSe}, 2}=200 \mathrm{~nm}, t_{\mathrm{Al}}=200 \mathrm{~nm}, d_{G}=100 \mathrm{~nm}, \Lambda_{G}=3.14 \mu \mathrm{m}$.

conditions, the waveguide cavity was optimized by varying the cavity width $\left(w_{\text {cavity }}\right)$ and cavity groove periodicity $\left(\Lambda_{C}\right)$ simultaneously. When the cavity width is tuned to the grating resonance, the bandwidth of the transmission peak can further be narrowed. The number of grooves in the cavity grating is low enough that it can be thought of as a set of scatterers, coupling the light downward out of the cavity. This decreases the strength of the relationship between the coupling efficiency of the cavity and the cavity grating periodicity.
As seen in Figure 4(a), the second-order input grating peak is suppressed after the introduction of an optimized waveguide cavity and the main resonance peak is enhanced. The second-order peak changed from $4.5 \%$ to $1.0 \%$ of the input power for the $11 \mu \mathrm{m}$ resonance. The first-order peak was enhanced after the introduction of the main cavity. The Q-factor increased from 108 to 150, narrowing the FWHM from $0.10 \mu \mathrm{m}$ to $0.07 \mu \mathrm{m}$. The efficiency also increased from $6.8 \%$ to $7.4 \%$. Figure 4 (b) shows how the transmission and $Q$-factor vary near a resonance. There is a tradeoff between the two metrics. The $Q$-factor reaches a maximum at a cavity width of about $26.8 \mu \mathrm{m}$, but the peak transmission reaches a maximum at $25.8 \mu \mathrm{m}$.

Figure 5 shows the time average power flow of the optimized $11 \mu \mathrm{m}$ structure at resonance. Above the input grating, the power entering the cavity is larger than the power being lost out of the sides in contrast to the equal power flow of the simple input grating. The power coupled into the waveguide from the two gratings interferes destructively on the outer portion of the structure, coupling more power into the cavity. Inside the cavity the germanium layer acts as a purely dielectric waveguide evanescently coupled to a surface plasmon waveguide (the metal cap) with the ZnSe-1 thickness controlling the coupling strength. Substantial interaction with the metal cap is obvious. The cavity grating serves to couple the power out of the waveguide in the downward direction. This structure is a band-pass filter which concentrates a narrow spectral band of infrared light from a large collection area $(524 \mu \mathrm{m})$ into a small transmission area $(22 \mu \mathrm{m})$. 


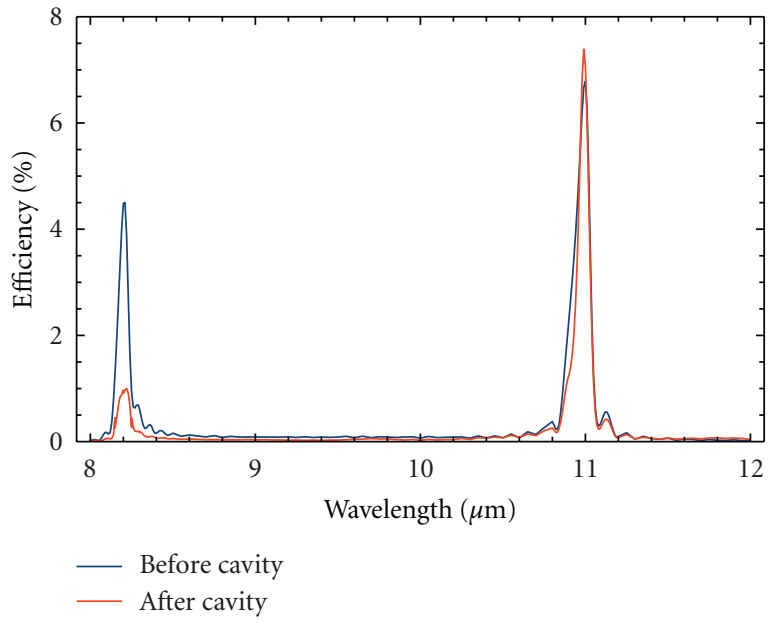

(a)

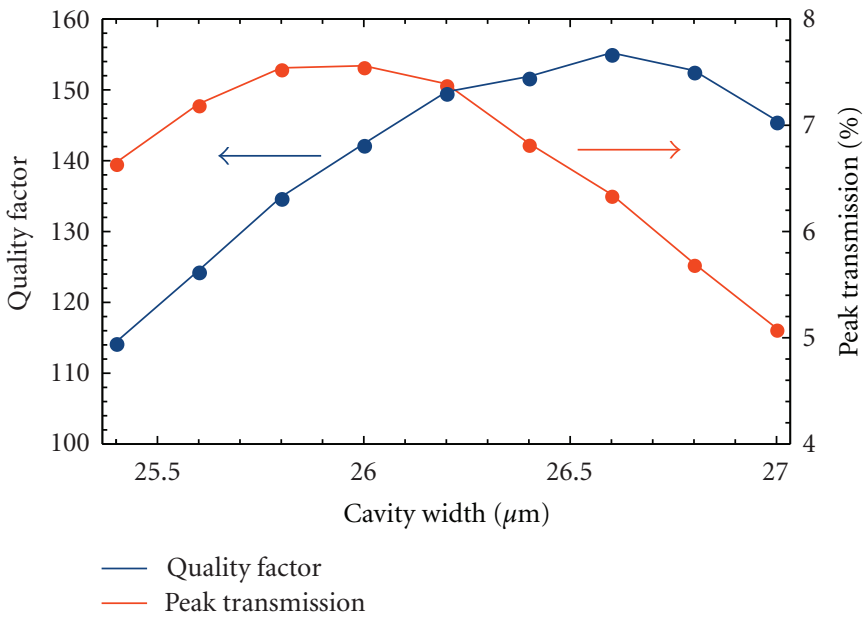

(b)

Figure 4: (a) Spectra before and after including a $26.2 \mu \mathrm{m}$ waveguide cavity. Coupling efficiency into the waveguide is plotted for "before cavity" and transmission is plotted for "after cavity." (b) Dependency of quality factor and peak transmission on the waveguide cavity width. Parameters used: grating parameters same as above, $w_{\text {cap }}=26.2 \mu \mathrm{m}, \Lambda_{C}=4.4 \mu \mathrm{m}, d_{C}=500 \mathrm{~nm}, t_{\mathrm{ZnSe}, 1}=1.1 \mu \mathrm{m}, t_{\text {cap }}=100 \mathrm{~nm}, N_{C}=4$.

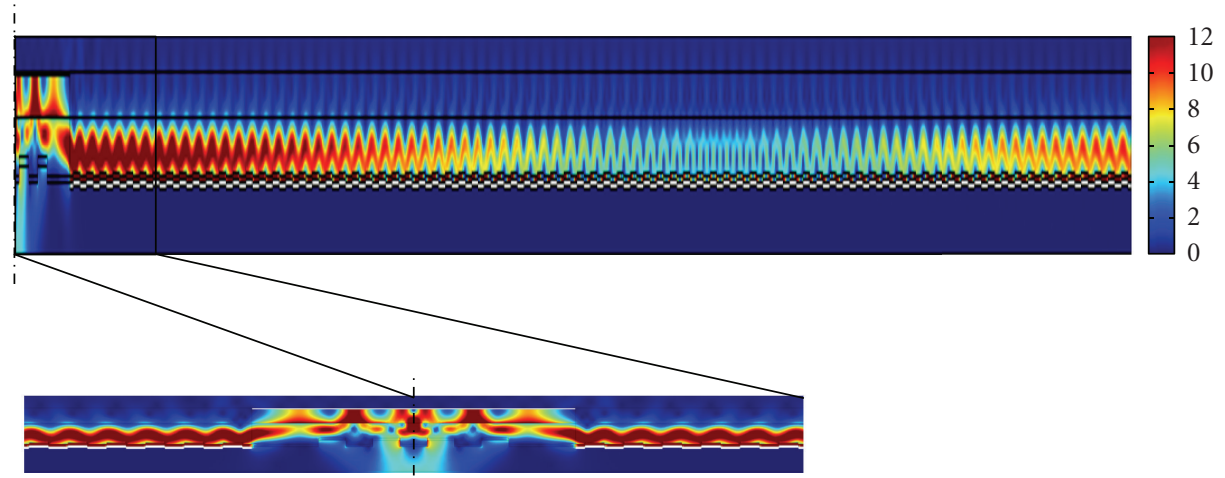

FIGURE 5: Power flow after introducing an optimized waveguide cavity. The aspect ratio of the lower picture is accurate, but the upper picture was stretched vertically by $2 \mathrm{x}$ for visibility. The color scale is power flow divided by incident power per unit area. Parameters used for this field plot: grating parameters same as above, $w_{\text {cap }}=26.2 \mu \mathrm{m}, \Lambda_{C}=4.4 \mu \mathrm{m}, d_{C}=500 \mathrm{~nm}, t_{\mathrm{ZnSe}, 1}=1.1 \mu \mathrm{m}, t_{\text {cap }}=100 \mathrm{~nm}, N_{C}=4, \lambda_{0}=11 \mu \mathrm{m}$.

3.3. Microbolometer Cavity Optimization. An array of microbolometers was placed below the filter with a back reflector a fixed $2 \mu \mathrm{m}$ below the pixels representing a commercially available focal plane array. The separation between the back reflector and the filter was adjusted to find the optimal vertical distance, with $h_{\mathrm{MC}}$ being the only free parameter. This was varied while calculating the power absorbed in the microbolometer array for each of the optimized transmission filters with different resonant wavelengths. The absorbed power was summed over all the microbolometers in the array. Figure 6(a) shows the peak behavior of our three filters as $h_{\mathrm{MC}}$ is varied. From this, a single value for $h_{\mathrm{MC}}$ was chosen to be $2.6 \mu \mathrm{m}$. At this value of $h_{\mathrm{MC}}$, the region where the microbolometer and input grating overlap acts as a waveguide. At small values of $h_{\mathrm{MC}}$, less light couples into this region, and at values larger than $2.6 \mu \mathrm{m}$, more light escapes between the microbolometer pixels. Figure 6(b) shows the absorption spectra for the three filters with $h_{\mathrm{MC}}=2.6 \mu \mathrm{m}$.
Figure 7 shows the power flow in the optimized $11 \mu \mathrm{m}$ transmission filter together with the microbolometer array. To summarize, input gratings couple power into the waveguide, which passes through the waveguide cavity, and is redirected to the microbolometer array. In the top image, the microbolometers (one centered under the cavity and parts of two others extending out of the model) are represented as white lines because they cannot be resolved at this scale. In contrast to the transmission filter alone where power beams from the center of the cavity, a closely spaced microbolometer results in power traveling along the microbolometer-vacuum-aluminum slot waveguide formed under the input gratings. The middle image provides a blowup of the power flow in this region with the microbolometer represented as a white bar. About $0.4 \%$ of the incident power leaks past the microbolometer centered under the cavity but is absorbed by its nearest neighbor. The final blowup shown at the bottom of Figure 7 shows the ohmic heating inside the microbolometer 


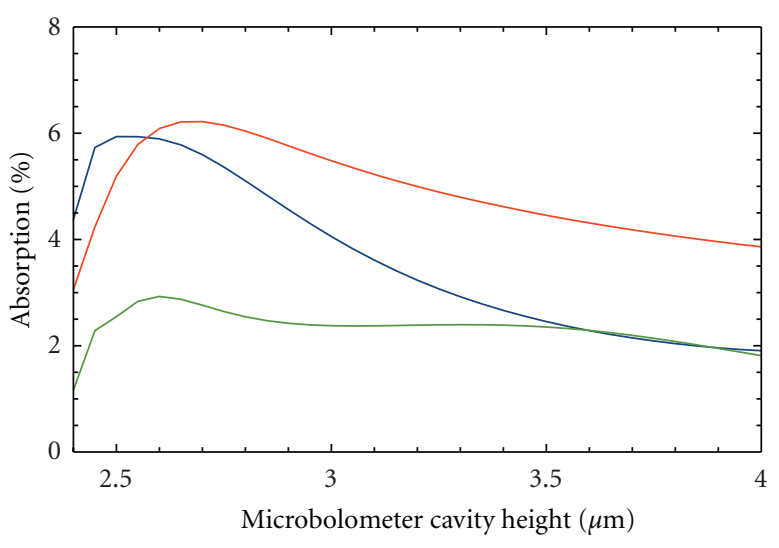

(a)

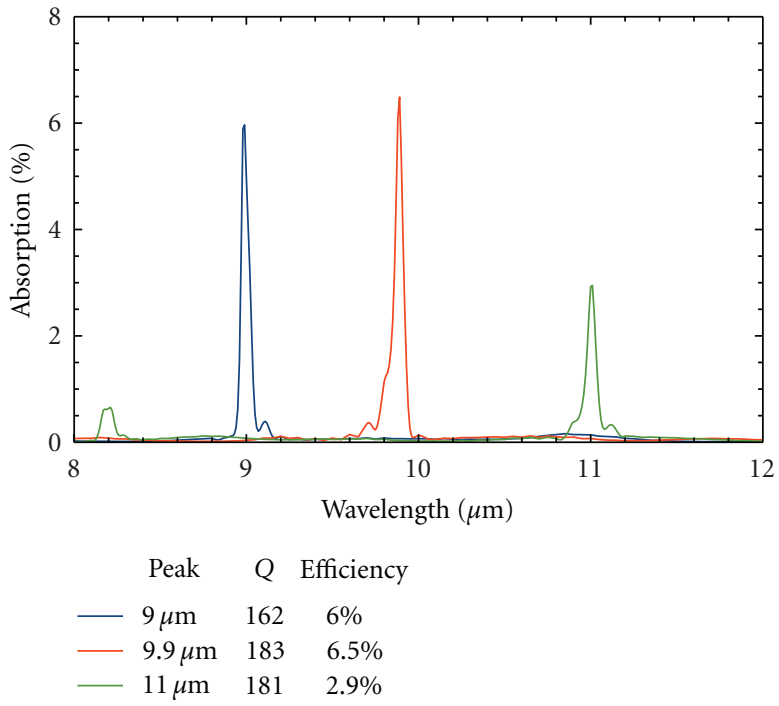

(b)

Figure 6: (a) Relative power absorbed in the microbolometer as a function of $h_{\mathrm{MC}}$ for the 9, 9.9, and $11 \mu \mathrm{m}$ filters. (b) Spectral performance for a microbolometer cavity height of $2.6 \mu \mathrm{m}$. Note that the legend is used for both (a) and (b).

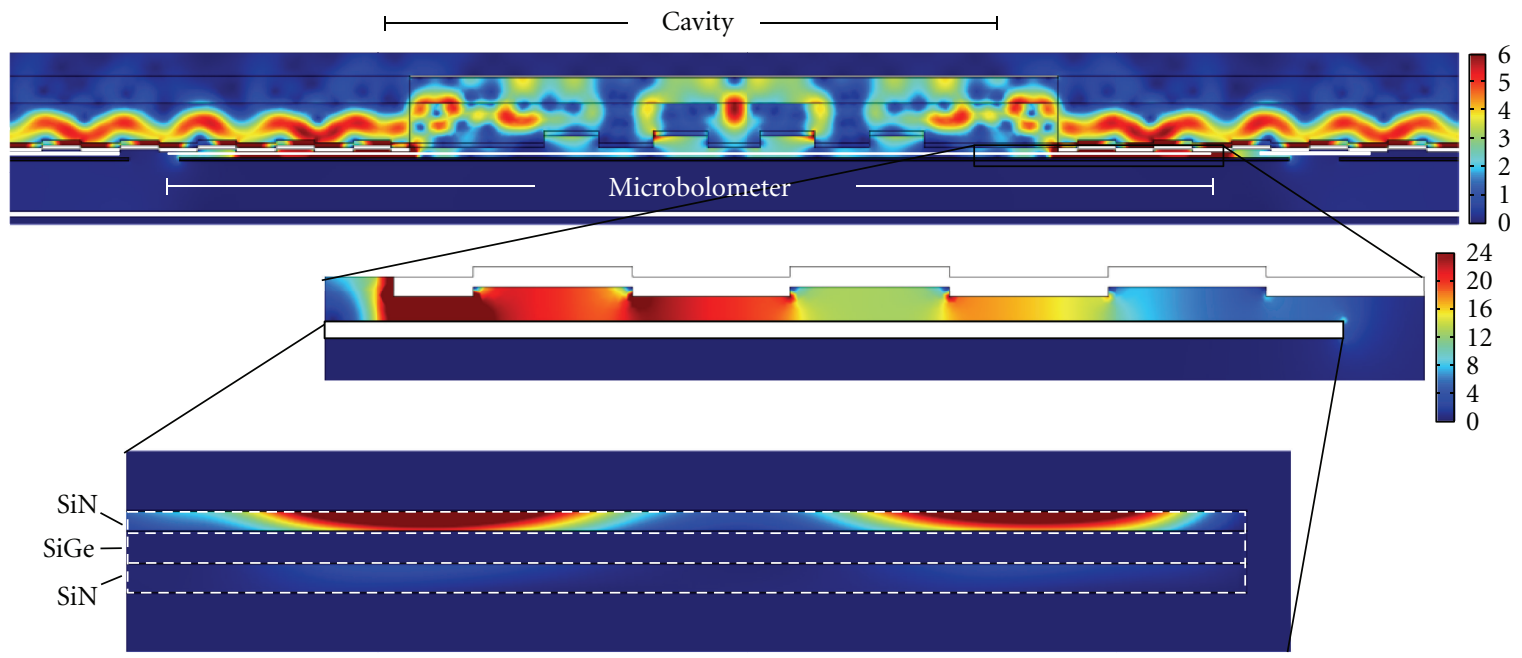

Figure 7: Power flow of the $11 \mu \mathrm{m}$ filter after optimizing $h_{\mathrm{MC}}$. First inset is a power flow plot of the overlap region on a different color scale. Second inset is a plot of ohmic heating in the microbolometer (aspect ratio is expanded vertically by $5 \mathrm{x}$ for better visibility).

element. This demonstrates that essentially all of the power is absorbed in the upper SiN layer of the pixel membrane. (SiN has an absorption band that covers all of the LWIR.)

3.4. Discussion. The microfilters described here were designed with a primary criterion of FWHM $<100 \mathrm{~nm}(Q>100$ for $10 \mu \mathrm{m}$ resonance) for compatibility with atmospheric pressure absorption spectroscopy. This was obtained through a combination of a narrow resonance from the input coupling grating combined with additional narrowing from the cavity resonance. For the input gratings, the resonance $Q$ increases with the number of grooves until it becomes limited by loss. There are two fundamental loss mechanisms captured by the modeling. One is ohmic losses in the metal due to the surface plasmon characteristic of the waveguide, with the strength adjustable through the $\mathrm{ZnSe}-2$ spacer layer. The second loss mechanism comes from the fact that a grating that couples normally incident light into a waveguide will also couple light traveling down the waveguide back out into free space. There will be an optimum grating length for maximum power in the waveguide that depends on the scattering strength of an individual groove: lower scattering strength allows gratings with more grooves. The relatively low transmission efficiencies of the designs presented here are a direct result of this tradeoff and our choice of having input coupling gratings with $Q>100$. Higher transmission efficiency can be obtained by allowing a higher line width in the coupling grating. 
TABLE 1

\begin{tabular}{|c|c|c|}
\hline \multicolumn{3}{|c|}{ Germanium } \\
\hline Wavelength $(\mu \mathrm{m})$ & $\epsilon_{1}$ & $\epsilon_{2}$ \\
\hline 1.6599 & 19.010 & $2.0249 \times 10^{-7}$ \\
\hline 1.6847 & 18.923 & $2.1484 \times 10^{-7}$ \\
\hline 1.708 & 18.836 & $2.2699 \times 10^{-7}$ \\
\hline 1.7319 & 18.749 & $2.3870 \times 10^{-7}$ \\
\hline 1.759 & 18.662 & $2.5397 \times 10^{-7}$ \\
\hline 1.7881 & 18.576 & $2.7230 \times 10^{-7}$ \\
\hline 1.8194 & 18.490 & $2.9136 \times 10^{-7}$ \\
\hline 1.8546 & 18.404 & $3.1404 \times 10^{-7}$ \\
\hline 1.8911 & 18.318 & $3.4008 \times 10^{-7}$ \\
\hline 1.932 & 18.233 & $3.7026 \times 10^{-7}$ \\
\hline 1.9776 & 18.148 & $4.0761 \times 10^{-7}$ \\
\hline 2.0287 & 18.063 & $4.5009 \times 10^{-7}$ \\
\hline 2.0858 & 17.978 & $5.0390 \times 10^{-7}$ \\
\hline 2.1516 & 17.893 & $5.6782 \times 10^{-7}$ \\
\hline 2.2236 & 17.808 & $6.4816 \times 10^{-7}$ \\
\hline 2.3067 & 17.724 & $7.5116 \times 10^{-7}$ \\
\hline 2.4073 & 17.640 & $8.8826 \times 10^{-7}$ \\
\hline 2.5221 & 17.556 & $1.0697 \times 10^{-6}$ \\
\hline 2.6592 & 17.472 & $1.3162 \times 10^{-6}$ \\
\hline 2.8429 & 17.389 & $1.7170 \times 10^{-6}$ \\
\hline 3.0646 & 17.306 & $2.3090 \times 10^{-6}$ \\
\hline 3.3584 & 17.222 & $3.3140 \times 10^{-6}$ \\
\hline 3.7684 & 17.140 & $5.2143 \times 10^{-6}$ \\
\hline 4.387 & 17.057 & $9.4583 \times 10^{-6}$ \\
\hline 5.3902 & 16.974 & $2.0995 \times 10^{-5}$ \\
\hline 6.2475 & 16.933 & $3.6862 \times 10^{-5}$ \\
\hline 7.0839 & 16.892 & $5.9312 \times 10^{-5}$ \\
\hline 8.0021 & 16.862 & $9.3486 \times 10^{-5}$ \\
\hline 9.0024 & 16.830 & $1.4333 \times 10^{-4}$ \\
\hline 9.6026 & 16.810 & $1.8172 \times 10^{-4}$ \\
\hline 10.01 & 16.798 & $2.1033 \times 10^{-4}$ \\
\hline 11.033 & 16.768 & $2.9662 \times 10^{-4}$ \\
\hline 12.405 & 16.728 & $4.4412 \times 10^{-4}$ \\
\hline
\end{tabular}

Additional gains in transmission efficiency can be obtained by terminating the outer edge of the input gratings with an appropriately spaced high-reflectivity mirror to reflect light which escapes in the waveguide away from the structure back toward the cavity.

The waveguide cavity provides additional improvement in the $Q$, but the amount is limited in practice. In general, a longer cavity will have a higher $Q$ provided propagation loss is minimal. However, this design leads to loss in the cavity through the output coupling grating in order to have transmission, which limits the cavity $Q$. The metal cap that blocks direct transmission through the cavity acts as an evanescently coupled surface plasmon waveguide, so there is also some ohmic loss here. The cap also sets a limit on the maximum transmission efficiency since any light directly incident on
TABLE 2

\begin{tabular}{lcc}
\hline & Silicon-germanium & \\
Wavelength $(\mu \mathrm{m})$ & $\epsilon_{1}$ & $\epsilon_{2}$ \\
\hline 1.6082 & 12.2183 & 0.1010 \\
1.7056 & 12.1366 & 0.1071 \\
1.8053 & 12.0684 & 0.1134 \\
1.9062 & 12.0101 & 0.1197 \\
2.0066 & 11.9616 & 0.1260 \\
2.2045 & 11.8855 & 0.1385 \\
2.4093 & 11.8269 & 0.1513 \\
2.6029 & 11.7842 & 0.1635 \\
2.8057 & 11.7485 & 0.1762 \\
3.0005 & 11.7216 & 0.1885 \\
3.2085 & 11.6976 & 0.2015 \\
3.3933 & 11.6804 & 0.2131 \\
3.6006 & 11.6639 & 0.2261 \\
3.8124 & 11.6501 & 0.2395 \\
4.0007 & 11.6397 & 0.2513 \\
4.2085 & 11.6300 & 0.2643 \\
4.4089 & 11.6217 & 0.2769 \\
4.6294 & 11.6133 & 0.2908 \\
4.8008 & 11.6077 & 0.3015 \\
5.0241 & 11.6021 & 0.3156 \\
5.4925 & 11.5915 & 0.3450 \\
6.0010 & 11.5821 & 0.3769 \\
6.5466 & 11.5754 & 0.4112 \\
7.0447 & 11.5701 & 0.4425 \\
7.5362 & 11.5654 & 0.5353 \\
8.0014 & 11.5627 & \\
8.5278 & 11.5592 & \\
9.0016 & 11.5572 & \\
9.9710 & 11.5536 & \\
10.9850 & & \\
12.0021 & 1.5484 & \\
& & \\
\hline
\end{tabular}

the cap is lost. In our design optimization, the cavity width was restricted to be less than $45 \mu \mathrm{m}$ so that it would cover only a single microbolometer pixel. The incorporation of the microbolometer impacts both $Q$ and transmission efficiency in a complicated manner, with $Q$ decreasing for the $9 \mu \mathrm{m}$ design but increasing for the 9.9 and $11 \mu \mathrm{m}$ designs. Adding the microbolometer roughly doubled the transmission efficiency of the $9 \mu \mathrm{m}$ design, slightly decreased the efficiency of the $9.9 \mu \mathrm{m}$ design, and cut the $11 \mu \mathrm{m}$ efficiency in half. Strong coupling between the various geometric parameters makes isolating the cause of this behavior difficult. But this coupling does provide the potential of substantial performance improvements through simultaneous multiparameter optimization.

The dimensions of the microfilters presented here ( $\sim 500 \mu \mathrm{m}$ average length) are compatible with fabricating a 100 wavelength channel spectrometer on a chip using a commercial $17 \mu \mathrm{m}$ pixel pitch $320 \times 240$ focal plane 
TABLE 3

\begin{tabular}{|c|c|c|}
\hline \multicolumn{3}{|c|}{ Silicon nitride } \\
\hline Wavelength $(\mu \mathrm{m})$ & $\epsilon_{1}$ & $\epsilon_{2}$ \\
\hline 1.5693 & 3.8385 & 0.0000 \\
\hline 2.5024 & 3.7547 & 0.0000 \\
\hline 3.5033 & 3.6165 & 0.0000 \\
\hline 4.5008 & 3.4181 & 0.0000 \\
\hline 5.2267 & 3.2249 & 0.0000 \\
\hline 6.0010 & 2.9546 & 0.0000 \\
\hline 6.4170 & 2.7692 & 0.0000 \\
\hline 6.8223 & 2.5456 & 0.0000 \\
\hline 7.2012 & 2.2698 & 0.0015 \\
\hline 7.4496 & 2.0195 & 0.0173 \\
\hline 7.6249 & 1.7896 & 0.0665 \\
\hline 7.8086 & 1.5125 & 0.2002 \\
\hline 8.0014 & 1.2459 & 0.4723 \\
\hline 8.1014 & 1.1533 & 0.6623 \\
\hline 8.2040 & 1.1094 & 0.8740 \\
\hline 8.3092 & 1.1250 & 1.0856 \\
\hline 8.4171 & 1.1988 & 1.2693 \\
\hline 8.5278 & 1.3155 & 1.3977 \\
\hline 8.6415 & 1.4459 & 1.4517 \\
\hline 8.7583 & 1.5515 & 1.4279 \\
\hline 8.8782 & 1.5936 & 1.3428 \\
\hline 9.0016 & 1.5446 & 1.2327 \\
\hline 9.1283 & 1.3981 & 1.1455 \\
\hline 9.2587 & 1.1748 & 1.1269 \\
\hline 9.3929 & 0.9180 & 1.2027 \\
\hline 9.5311 & 0.6746 & 1.3681 \\
\hline 9.6733 & 0.4724 & 1.5915 \\
\hline 9.8199 & 0.3052 & 1.8378 \\
\hline 9.9710 & 0.1420 & 2.0966 \\
\hline 10.1268 & -0.0460 & 2.3956 \\
\hline 10.2875 & -0.2536 & 2.7883 \\
\hline 10.4535 & -0.4308 & 3.3210 \\
\hline 10.6248 & -0.4953 & 4.0036 \\
\hline 10.8019 & -0.3635 & 4.7953 \\
\hline 10.9850 & 0.0218 & 5.6136 \\
\hline 11.1744 & 0.6732 & 6.3532 \\
\hline 11.3704 & 1.5576 & 6.9128 \\
\hline 11.5734 & 2.6011 & 7.2128 \\
\hline 11.7838 & 3.7055 & 7.2086 \\
\hline 12.0021 & 4.7635 & 6.8976 \\
\hline
\end{tabular}

array. The filters can be fabricated on the inside of the $\mathrm{Si}$ wafer lid used during wafer level vacuum packaging [13], representing only a marginal increase in fabrication complexity. Our design used a ZnSe substrate for convenience, but this can readily be adapted to Si substrates by adding an additional $\mathrm{ZnSe}$ spacer layer between the substrate and metal cap. Achieving the small pixel to filter separation could be difficult with the filter on the lid, but the design can be optimized for larger separations if necessary. Alternatively, a multilevel MEMS fabrication approach could be implemented. The filter itself requires only standard photolithography, thin film processes, and dielectric materials commonly used for LWIR optical coatings. Filter fabrication should therefore be straight forward.

\section{Conclusion}

The design of a plasmonic narrow band-pass LWIR microfilter coupled to a microbolometer sensor array was presented. The filter can be tuned to select any desired wavelength in the full LWIR atmospheric transmission range by simple adjustment of horizontal parameters in a common set of lithography steps, greatly simplifying synthesis complexity relative to existing approaches. Bandwidths below $61 \mathrm{~nm}$ FWHM are predicted across the tested range. Using appropriate highand low-index materials, this design can be adapted for operation in the visible, infrared, or into the $\mathrm{THz}$ regions.

\section{Appendix}

\section{Tables of Ellipsometrically Measured Optical Constants}

See Tables 1, 2, and 3.

\section{Acknowledgments}

This material is based upon work supported by the U.S. Army, Edgewood Chemical and Biological Center under Contract no. W911SR-10-C-0086.

\section{References}

[1] C. Gittins, W. Marinelli, and J. Jensen, "Remote characterization of chemical vapor plumes by LWIR imaging fabry-perot spectrometry," in Proceedings of the 5th Joint Conference on Standoff Detection for Chemical and Biological Defense, pp. 428, Williamsburg, Va, USA, 2001.

[2] J. T. Daly, A. Bodkin, W. Schneller et al., "Tunable narrowband filter for LWIR hyperspectral imaging," in Proceedings of the Photodetectors: Materials and Devices V, Proceedings of SPIE, pp. 104-115, January 2000.

[3] R. Bhan, R. Saxena, C. Jalwania, and S. Lomash, "Uncooled infrared microbolometer arrays and their characterisation techniques," Defence Science Journal, vol. 59, no. 6, pp. 580-589, 2009.

[4] J. J. Talghader, A. S. Gawarikar, and R. P. Shea, "Beyond the blackbody radiation limit: high-sensitivity thermal detectors," in Proceedings of the Infrared Technology and Applications XXXVI, vol. 7660 of Proceedings of SPIE, p. 766011, Orlando, Fla, USA, April 2010.

[5] A. S. Weling and P. F. Henning, "Antenna-coupled microbolometers for multispectral infrared imaging," in Proceedings of the Infrared Technology and Applications XXXII, vol. 6206 of Proceedings of SPIE, p. 62061F, April 2006.

[6] S. W. Han and D. P. Neikirk, "Design of infrared wavelengthselective microbolometers using planar multimode detectors," 
in Proceedings of the Smart Sensors, Actuators, and MEMS II, Proceedings of SPIE, pp. 549-557, May 2005.

[7] F. González, J. Porter, and G. Boreman, "Antenna-coupled infrared focal plane array," Microwave and Optical Technology Letters, vol. 48, no. 1, pp. 165-166, 2006.

[8] T. Maier and H. Brueckl, "Multispectral microbolometers for the midinfrared," Optics Letters, vol. 35, no. 22, pp. 3766-3768, 2010.

[9] T. Maier and H. Brückl, "Wavelength-tunable microbolometers with metamaterial absorbers," Optics Letters, vol. 34, no. 19, pp. 3012-3014, 2009.

[10] N. I. Landy, S. Sajuyigbe, J. J. Mock, D. R. Smith, and W. J. Padilla, "Perfect metamaterial absorber," Physical Review Letters, vol. 100, no. 20, Article ID 207402, 2008.

[11] Y. Avitzour, Y. A. Urzhumov, and G. Shvets, "Wide-angle infrared absorber based on a negative-index plasmonic metamaterial," Physical Review B, vol. 79, no. 4, Article ID 045131, 2009.

[12] Y. Wang, B. Potter, and J. Talghader, "Coupled absorption filters for thermal detectors," Optics Letters, vol. 31, no. 13, pp. 1945-1947, 2006.

[13] A. Syllaios and P. Chahal, "Integrated spectroscopic microbolometer with microfilter arrays," U.S. Patent No. 6,998,613, 2004.

[14] Z. Jaksic, R. Petrovic, D. Randjelovic et al., "Integrated multicolor detector utilizing 1-D photonic bandgap filter with wedge-shaped defect," in Proceedings of the 1999 Design, Test, and Microfabrication of MEMS and MOEMS, Proceedings of SPIE, pp. 611-619, April 1999.

[15] Y. Wang, B. Potter, M. Sutton, R. Supino, and J. Talghader, "Step-wise tunable microbolometer long-wavelength infrared filter," in 13th International Conference on Solid-State Sensors and Actuators and Microsystems, pp. 1006-1009, June 2005.

[16] Q. Cheng, S. Paradis, T. Bui, and M. Almasri, "Design of dualband uncooled infrared microbolometer," IEEE Sensors Journal, vol. 11, no. 1, pp. 167-175, 2011.

[17] R. P. Shea, A. S. Gawarikar, and J. J. Talghader, "Midwave thermal infrared detection using semiconductor selective absorption," Optics Express, vol. 18, no. 22, pp. 22833-22841, 2010.

[18] Z. Yu, G. Veronis, S. Fan, and M. L. Brongersma, "Design of midinfrared photodetectors enhanced by surface plasmons on grating structures," Applied Physics Letters, vol. 89, no. 15, Article ID 151116, 2006.

[19] P. D. Flammer, I. C. Schick, R. T. Collins, and R. E. Hollingsworth, "Interference and resonant cavity effects explain enhanced transmission through subwavelength apertures in thin metal films," Optics Express, vol. 15, no. 13, pp. 7984-7993, 2007.

[20] S. Ajmera, A. Syllaios, G. Tyber, M. Taylor, and R. Hollingsworth, "Amorphous silicon thin-films for uncooled infrared microbolometer sensors," in Proceedings of the Infrared Technology and Applications XXXVI, vol. 7660 of Proceedings of SPIE, p. 766012, Orlando, Fla, USA, 2010.

[21] C. M. Hanson, S. K. Ajmera, J. Brady et al., "Small pixel a-Si/ a-SiGe bolometer focal plane array technology at L-3 communications," in Proceedings of the Infrared Technology and Applications XXXVI, vol. 7660 of Proceedings of SPIE, p. 76600R, Orlando, Fla, USA, 2010.

[22] J. Jin, The Finite Element Method in Electromagnetics, Wiley, 2nd edition, 2002.

[23] E. Palik, Handbook of Optical Constants of Solids, Academic Press, 1985.

[24] M. Bass, G. Li, and E. V. Stryland, Handbook of Optics, vol. 4, McGraw-Hill, 3rd edition, 2009.
[25] A. D. Rakic, "Algorithm for the determination of intrinsic optical constants of metal films: application to aluminum," Applied Optics, vol. 34, no. 22, pp. 4755-4767, 1995. 

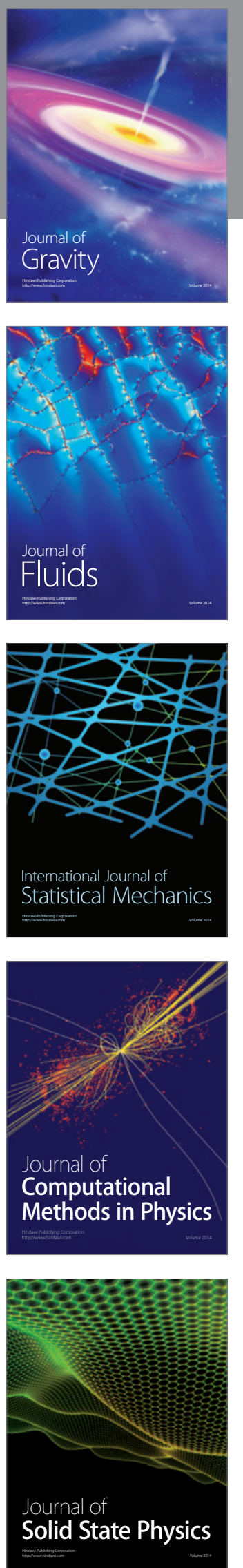

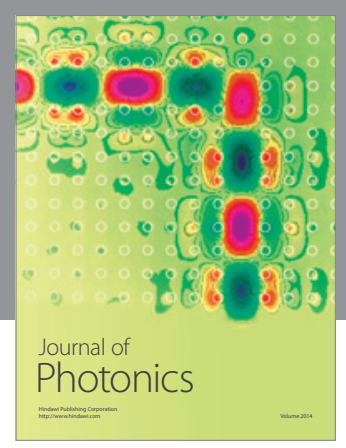

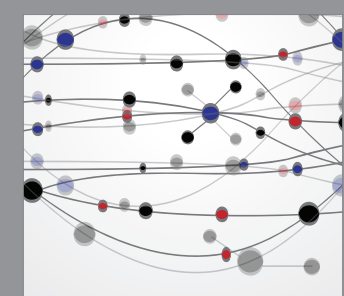

The Scientific World Journal
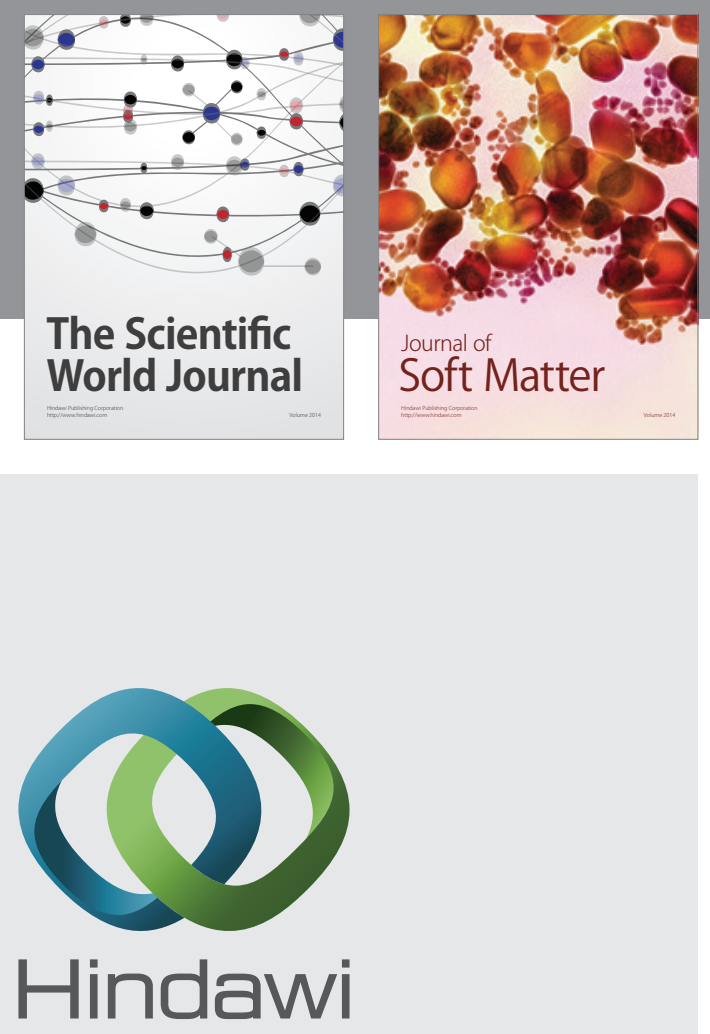

Submit your manuscripts at

http://www.hindawi.com
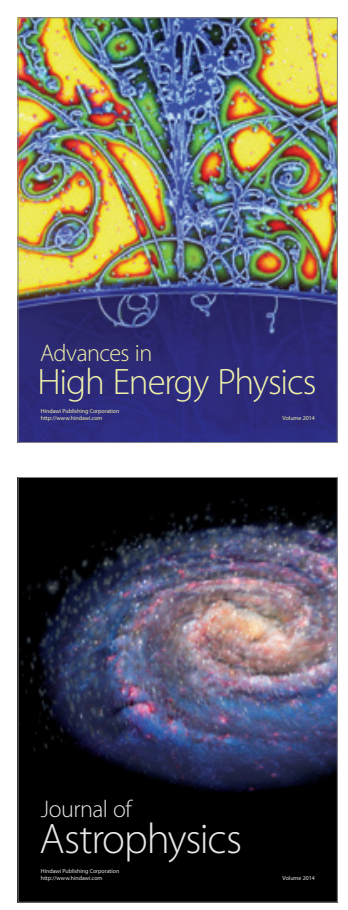
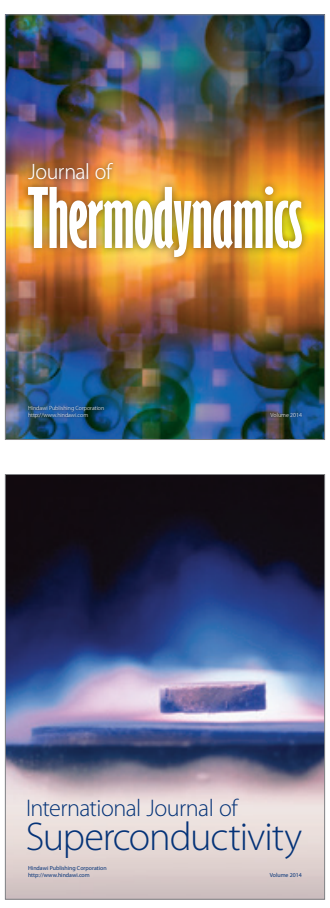
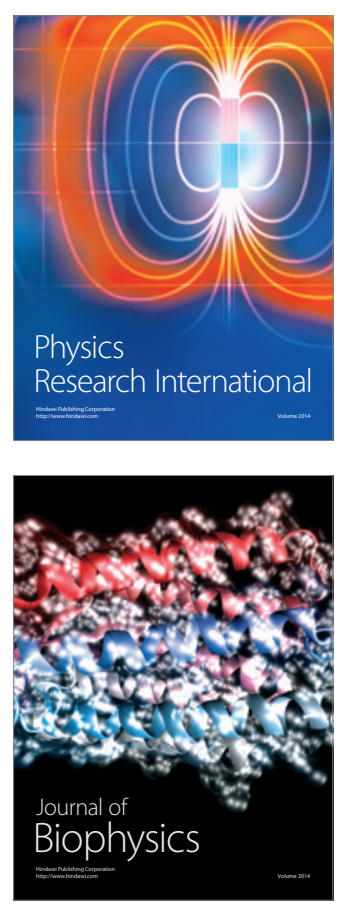
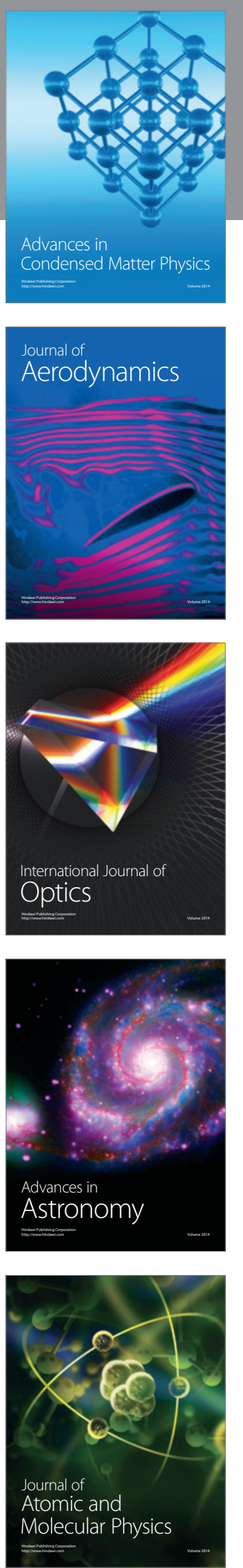